\title{
Using Kohonen's Self-Organizing Map for Clustering in Sensor Networks
}

\author{
Chiranjib Patra \\ Calcutta Institute of Engineering and \\ Management. \\ 24/1A Chandi Ghosh Rd. \\ Tollygunge ,kolkata-700040
}

\begin{abstract}
Clustering is a technique that can be used to classify objects (e.g. individuals, quadrates, species etc). While Kohonen's SelfOrganizing Map (SOM) networks have been successfully applied as a classification tool to various problem domains, including Mobile Ad-hoc networks, sensor networks, robot control and medical diagnosis, its potential as a robust substitute for clustering analysis remains relatively unresearched. In this paper, SOM is used to cluster the sensor nodes dispersed in nonhomogeneous space so that efficient transmit power management of the nodes can be made.
\end{abstract}

\section{Keywords}

Sensor Networks, Self organizing maps, cluster Analysis

\section{INTRODUCTION}

Economizing on power consumption is very important aspect in the field of sensor network research. Clustering can be of great importance to divide the network by some disjoint clusters as the result the sizes of the routing tables are reduced, redundancy of exchanged messages and extend the life time of the network [1].

In case of homogeneously distributed nodes in network it is possible to solve problems very easily by controlling with minimum transmission power which connects the network. However when nodes in a network are non homogeneously distributed the distances affect the battery life and traffic transmission becomes inefficient [4].

Kohonen's self -organizing maps (SOMs) are special type of artificial neural network, which provides projection of multidimensional data into one-, two- or three- dimensional space. It was designed for clustering, visualization and abstraction [2].The fundamental of SOM is the soft competition between the nodes in the output layer; not only one node (the winner) but also the neighbors are updated .The Kohonen's network can recognize clusters of data and can relate clusters of data and relate similar classes to one another. SOM has only two layers: input and the output layer. The input layer is one dimensional while the output layer consists of radial units typically organized in two dimensions. SOM adapt to the training data in a way such that a high dimension input space is reflected to two dimensional grid generated Kohonen's map [3].
This paper is organized as follows: section 2 describes proposed clustering algorithm. Section 3 describes clustering characteristics for transmit power control. Finally, section 4 concludes this paper and discusses future work.

\section{Clustering Algorithm}

The proposed technique of clustering of sensor nodes generates clusters with determining power level by Kohonen's self organizing map in non-homogeneously spatial network.

\subsection{Clustering algorithm}

The algorithm can be described as follows:

The SOM defines a mapping of input data spanned by $\mathrm{x}_{1}, \mathrm{x}_{2}, \mathrm{x}_{3}, \ldots \ldots, \mathrm{x}_{\mathrm{n}}$ onto one or two dimension array of nodes. Each node of the map is defined by a vector $\mathrm{w}_{\mathrm{ij}}$ whose elements are adjusted during training. The basic training algorithm is quite simple.

- $\quad$ Select an object from the training set.

- Find the node which is close to the selected data (i.e. the distance between $\mathrm{w}_{\mathrm{ij}}$ and the training data is minimum.)

- Adjust the weight vectors of the closest node and the nodes around it in a way that $\mathrm{w}_{\mathrm{ij}}$ moves towards the training data.

- Repeat from step 1 for a fixed number of repetitions.

The amount of adjustment in step 3 as well as the range of neighborhood decreases during training. This ensures that there were coarse adjustments in the first phase of the training, while fine tuning occurs at the end [2].

The above theory can be utilized to create a learning algorithm for sensor networks.

\section{Begin}

Step 1. Assign random variables to the connection weights $\mathrm{W}$ in the range $(0,1)$.

Step 2. Select input pattern from the row of an adjacency matrix of the network topology corresponding to a transmission power level. 
Step 3. Determine the winner output neuron.

Step 4. Perform a learning step affecting all neurons in neighborhood of the winner output neuron by updating the connection weights.

$\mathrm{W}_{\mathrm{j}}($ new $)=\mathrm{w}_{\mathrm{j}}($ old $)+\alpha \mathrm{h}_{\mathrm{t}}\left(\mathrm{I}-\mathrm{w}_{\mathrm{j}}(\right.$ old $\left.)\right)$

Where $\alpha$ is learning rate parameter, $\mathrm{I}$ is the input pattern.

Update $\alpha$ and $h_{t}$ and continue with step 2 until no noticeable changes in the connection weights are observed for all input patterns.

End

$\left[h_{t}=h_{0}(1-t / T)\right.$, where $h_{t}$ denotes the actual neighborhood size, $\mathrm{h}_{0}$ denotes the initial neighborhood size, $\mathrm{t}$ denotes the current learning epoch, and $\mathrm{T}$ denotes the total number of epochs to be done, where an epoch is the time taken for network to sweep through all the rows of adjacency matrix as an input data pattern once. ]

In general, clustering will lead to modified routing table which will include cluster id and transmitted power level. The upper threshold of power level for a cluster can be determined by manual setting the power required to communicate the fringe sensor with the centroid. This setting can be used as optimized power level for communication amongst cluster members.

\section{Simulation Details}

MATLAB was used to simulate clustering of the given sensor network. Our simulation included 50 node spread over $100 \mathrm{mX100m}$ field .Simulations show how randomly scattered sensors can condense to an orderly cluster depicted in the diagram below.
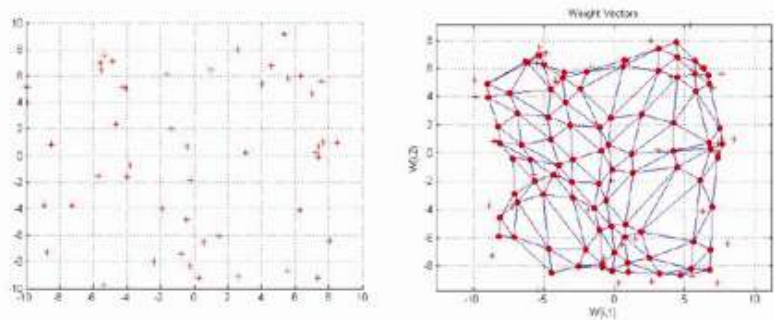

Fig-1 a. randomly distributed b. clustered using SOM
From fig 1 it's clear that randomly distributed sensors over a given topology can be clustered to provide efficient routing and power management for transmission.

\subsection{Implementation Details}

As per the algorithm the weights were random created from 0 to 1 then the input was taken as a set 2-dimensional coordinates in $\mathrm{x}$ and $\mathrm{y}$. Using the function newsom a new self-organizing map was created with following parameters

PR -- R x 2 matrix of min and max values for R input elements ,[-100 100;-100 100]

Di -- Size of ith layer dimension $[9,10]$

TFCN -- Topology function, default ='hextop'

DFCN -- Distance function, default ='linkdist'

OLR -- Ordering phase learning rate, default $=0.9$

OSTEPS -- Ordering phase steps, default $=1000$

TLR -- Tuning phase learning rate, default $=0.02$

TND -- Tuning phase neighborhood distance, default $=1$

For the calculation of epochs the neighborhood size $h_{0}=40$ was chosen

\section{Conclusions and Future Scope}

This paper discusses the clustering of the like sensors i.e. clustering with respect to transmission power level. Proper clustering at the ground level will improve routing capabilities and energy saving.

The algorithm discussed provides an efficient clustering method which requires to be proved by NS2 simulator.

\section{REFERENCES}

[1] Narayanswamy.S, Kawada.V, Srenivas.R.S, and Kumar.R.S: Power Control in Ad-Hoc networks: Theory, Architecture, Algorithm, and Implementation of the COMPOW protocol, European Wireless Conference,(2002)

[2] SDL component Suite,"Kohonen Network-Background Information", http://www.lohninger.com/helpsuite/kohonen network back ground information.htm.

[3] Simon Haykin, Neural Networks A comprehensive Foundation ,Pearson Education, New Delhi ,2007

[4] Kawadia.V, Kumar P.R.: power control and clustering Ad Hoc Networks. IEEE INFOCOM,(2003) 